\title{
Coretractable modules relative to a submodule
}

\section{Ali Reza Moniri Hamzekolaee, Yahya Talebi}

Abstract: Let $R$ be a ring and $M$ a right $R$-module. Let $N$ be a proper submodule of $M$. We say that $M$ is $N$-coretractable (or $M$ is coretractable relative to $N$ ) provided that, for every proper submodule $K$ of $M$ containing $N$, there is a nonzero homomorphism $f: M / K \rightarrow M$. We present some conditions that a module $M$ is coretractable if and only if $M$ is coretractable relative to a submodule $N$. We also provide some examples to illustrate special cases.

2010 MSC: 16D10, 16D40, 16D80

Keywords: Coretractable module, $N$-coretractable module

\section{Introduction}

Throughout this paper $R$ will denote an arbitrary associative ring with identity and all modules will be unitary right $R$-modules unless stated otherwise. Let $M$ be an $R$-module. We use $\operatorname{End}_{R}(M)$, $\operatorname{ann}_{r}(M)$ (in the case $M$ is a right $R$-module), $a n n_{l}(M)$ (in the case $M$ is a left $R$-module) to denote the ring of endomorphisms of $M$, the right annihilator in $R$ of $M$ and the left annihilator in $R$ of $M$, respectively. Let $M$ be a module and $K$ a submodule of $M$. Then $K$ is essential in $M$ denoted by $K \leq_{e} M$, if $L \cap K \neq 0$ for every nonzero submodule $L$ of $M$. Dually, $K$ is small in $M(K \ll M)$, in case $M=K+L$ implies that $L=M$. A submodule $N$ of $M$ is called supplement, if there is a submodule $K$ of $M$ such that $M=N+K$ and $N \cap K \ll N$. A module $M$ is called supplemented if every submodule of $M$ has a supplement in $M$. For any unexplained terminology we refer to [3], [9] and [11].

Khuri in [5] introduced the concept of a retractable module. Let $M$ be a module. Then $M$ is retractable in case for every nonzero submodule $N$ of $M$, there is a nonzero homomorphism $f: M \rightarrow N$, i.e $\operatorname{Hom}_{R}(M, N) \neq 0$. In the literature, there are some works about retractable modules (see [6, 12, 14]). Amini, Ershad and Sharif in [2] defined a dual notation namely coretractable modules. A module $M$ is coretractable provided that, $\operatorname{Hom}_{R}(M / N, M) \neq 0$ for every proper submodule $N$ of $M$. There are also some papers whose main subject is to study and investigate coretractable modules. We refer readers to $[1,4,13]$ for more information about coretractable modules.

Ali Reza Moniri Hamzekolaee (Corresponding Author), Yahya Talebi; Department of Mathematics, Faculty of Mathematical Sciences, University of Mazandaran, Babolsar, Iran (email: a.monirih@umz.ac.ir, talebi@umz.ac.ir). 
In [10], the author introduced a generalization of coretractable modules via the cosingular submodule. Following [10], a module $M$ is called $\bar{Z}(M)$-coretractable in case, for every proper submodule $N$ of $M$ containing $\bar{Z}(M)$, there is a nonzero homomorphism $f: M / N \rightarrow M$. It is proved in [10, Theorem 2.11] that a ring $R$ is $\bar{Z}\left(R_{R}\right)$-coretractable if and only if every finitely generated free right $R$-module $F$ is $\bar{Z}(F)$-coretractable. Also, a characterization of commutative semiperfect Kasch rings is presented via $\bar{Z}$-coretractablity ([10, Corollary 2.14]). Inspiring by [10], we are interested to study coretractablity of modules relative to their submodules. If in the definition of a coretractable module $M$, we fix a submodule $N$ and focus just on nonzero homomorphisms from $M / K$ to $M$ where $K \neq M$ contains $N$, we have a special generalization of coretractable modules. We may choose special submodules of a module $M$ such as $\operatorname{Soc}(M), \operatorname{Rad}(M)$ and some others. We present some necessary conditions to prove that when two concepts coretractable and coretractable relative to a submodule coincide. Among them, we show that for a small or a semisimple submodule $N$ of $M, M$ is coretractable if and only if $M$ is $N$-coretractable. It is also shown that if $M$ is $N$-coretractable and $N$ is coretractable, then $M$ is coretractable. For a right ideal $I$ of $R$, we show that $R_{R}$ is $I$-coretractable if and only if every simple right $R$-module that is annihilated by $I$, can be embedded in $R_{R}$. As a consequence, $R_{R}$ is coretractable if and only if $R$ is right Kasch.

\section{Coretractable modules relative to a submodule}

In this section we introduce a new generalization of coretractable modules via submodules.

Recall that a module $M$ is coretractable, in case for every proper submodule $N$ of $M$, there exists a nonzero homomorphism $f: M / N \rightarrow M$.

Definition 2.1. Let $M$ be a module and $N$ a proper submodule of $M$. We say $M$ is $N$-coretractable in case for every proper submodule $K$ of $M$ containing $N$, there is a nonzero homomorphism $f: M / K \rightarrow M$. Note that a module $M$ is coretractable if and only if $M$ is $\{0\}$-coretractable.

Let $M$ be a module and $N$ a proper submodule of $M$. It is not hard to verify that $M$ is $N$ coretractable if and only if for every proper essential submodule $K$ of $M$ containing $N$, there is a nonzero homomorphism from $M / K$ to $M$.

Note that if a module $M$ is $N$-coretractable, then for every submodule $T \subseteq N$, there is a nonzero homomorphism $g: M / T \rightarrow M$. In fact, if $M$ is $N$-coretractable, then for every submodule $T$ of $M$, either contained in $N$ or containing $N$, there will be a nonzero homomorphism from $M / T$ to $M$.

Recall from [7], a ring $R$ is right (left) Kasch in case every simple right (left) $R$-module can be embedded in $R_{R}\left({ }_{R} R\right)$. In [2, Theorem 2.14], the authors proved that $R$ is right Kasch if and only if $R_{R}$ is coretractable.

Let $R$ be a right Kasch ring which is not left perfect. Then by [4, Proposition 2.9], there is a right ideal $I$ of $R$ such that $R / I$ is not coretractable while $R_{R}$ is coretractable as $R$ is a Kasch ring (see also [4, Example 2.10]).

Lemma 2.2. (1) Let $N, K, N_{i}<M$. Let $M$ be $N$-coretractable. If $K \supseteq N$, then $M$ is $K$-coretractable. In particular, if $M$ is $N_{i}$-coretractable for each $i \in I$, then $M$ is $\left(\sum_{i \in I} N_{i}\right)$-coretractable.

(2) Let $M$ be $N$-coretractable. If $K \leq N$ such that $K$ contains no nonzero image of any endomorphism of $M$, then $M / K$ is $N / K$-coretractable. In a special case, if $M$ is $N$-coretractable such that for every $f \in \operatorname{End}(M), \operatorname{Imf} \nsubseteq \Phi$, then $M / N$ is coretractable (see [4, Proposition 2.11]).

Proof. (1) This is straightforward.

(2) Let $T / K$ be a proper submodule of $M / K$ containing $N / K$. Then $N \subseteq T \subset M$. Since $M$ is $N$-coretractable, there exists a nonzero homomorphism $g: M / T \rightarrow M$. Now define $h: \frac{M / K}{T / K} \rightarrow M / K$ by $h\left(x+K+\frac{T}{K}\right)=g(x+T)+K$ for every $x \in M$. If $\operatorname{Imh}=0$, then $\operatorname{Img} \subseteq K$. Now, $K$ contains 
the image of the endomorphism go $\pi$ of $M$ where $\pi: M \rightarrow M / T$ is the natural epimorphism, this gives a contradiction. Therefore, $M / K$ is $N / K$-coretractable.

Let $R$ be a right Noetherian ring and $M$ be a $N$-coretractable module where $N$ is a finitely generated proper submodule of $M$. Then by Lemma $2.2(2), M / N$ is coretractable (see [4, Corollary 2.13]).

Proposition 2.3. Let $M$ be a module and $K \leq N<M$. If $M / K$ is $N / K$-coretractable and $M / K$ can be embedded in $M$, then $M$ is $N$-coretractable. In particular, if $M=K \oplus K^{\prime}$ and $N$ is any submodule of $M$ such that $K^{\prime}$ is $\left(N \cap K^{\prime}\right)$-coretractable, then $M$ is $N$-coretractable.

Proof. Let $T$ be a proper submodule of $M$ containing $N$. Then $T / K$ is a proper submodule of $M / K$ containing $N / K$. By assumption, there is a nonzero homomorphism $g: \frac{M / K}{T / K} \cong M / T \rightarrow M / K$. There also exists a monomorphism $h: M / K \rightarrow M$. Now, the homomorphism $h o g: M / T \rightarrow M$ is the required one.

Corollary 2.4. Let $M$ be a module and $N<M$ such that $M / N$ is coretractable. If $M / N$ can be embedded in $M$, then $M$ is $N$-coretractable. In particular, if $M$ is supplemented with Rad $(M)$ a direct summand of $M$, then $M$ is $\operatorname{Rad}(M)$-coretractable.

Proof. This is a special case of Proposition 2.3. The last part follows from the fact that for a supplemented module $M$, the module $M / \operatorname{Rad}(M)$ is coretractable since $M / \operatorname{Rad}(M)$ is semisimple. In this case $M$ is $\operatorname{Rad}(M)$-coretractable.

Example 2.5. (1) Let $M$ be a coretractable module and $N<M$. Then $M$ is $N$-coretractable. In particular, every cogenerator $M$ in the category of right $R$-modules is coretractable relative to every $N<M$.

(2) Let $M$ be a module such that for every submodule $K$ of $M$ we have $M / K \cong M$. Then $M$ is coretractable relative to each $N<M$.

(3) Let $M$ be a module and $N<M$. If every proper submodule of $M$ containing $N$, is contained in a proper summand of $M$, then $M$ is $N$-coretractable.

(4) Let $M$ be an uniserial module. If $M$ is coretractable relative to a proper submodule $N$, then $M$ is coretractable.

The following introduces a $N$-coretractable module which is not coretractable. In fact, the class of relative coretractable modules properly contains the class of coretractable modules.

Example 2.6. Let $P$ be the set of all prime numbers and $M=\prod_{p \in P} \mathbb{Z}_{p}$ as an $\mathbb{Z}$-module. Take $N=$ $\{0\} \times \mathbb{Z}_{3} \times \mathbb{Z}_{5} \times \ldots$ which is a maximal submodule of $M$, since $M / N \cong \mathbb{Z}_{2}$. Consider $g: \mathbb{Z}_{2} \rightarrow M$ defined by $g(x)=(x, 0,0, \ldots)$. Then $g$ is a nonzero homomorphism indicating that $M$ is $N$-coretractable. Note that by [2, Example 2.9], $M$ is not a coretractable $\mathbb{Z}$-module.

Remark 2.7. Let $M$ be a module and $N<M$. If there is not a nonzero homomorphism from $M / N$ to $M$, then $M$ is not $N$-coretractable. For example, let $M$ be a nonsingular module and $N$ be a proper submodule of $M$ such that $M / N$ is singular. So there does not exist any nonzero homomorphism from $M / N$ to $M$. Now, $M$ is not $N$-coretractable (for example, $\mathbb{Z}$-modules $\mathbb{Q}$ and $\mathbb{Z}$ can not be $n \mathbb{Z}$-coretractable).

We shall consider some conditions under which the two concepts coretractable and $N$-coretractable coincide.

Lemma 2.8. Let $M$ be a module and $N<M$. In each of the following cases $M$ is $N$-coretractable if and only if $M$ is coretractable.

(1) $N$ is a small submodule of $M$.

(2) $N$ is a coretractable module. 
Proof. (1) Let $M$ be $N$-coretractable where $N \ll M$ and $K$ be a proper submodule of $M$. Since $N$ is small in $M$, we have $N+K \neq M$. Now since $M$ is $N$-coretractable, then there is a nonzero homomorphism $f: M /(N+K) \rightarrow M$. So that $\operatorname{Hom}_{R}(M / K, M) \neq 0$. It follows that $M$ is coretractable. The converse is clear.

(2) Let $K$ be a proper submodule of $M$. Then either $K+N \neq M$ or $K+N=M$. If $K+N \neq M$, then similarly to (1) we have $\operatorname{Hom}_{R}(M / K, M) \neq 0$. Now suppose that $K+N=M$. Then there is an isomorphism $h: M / K \rightarrow N /(N \cap K)$ induced from $M=N+K$. Since $N$ is coretractable, there is a nonzero homomorphism $g: N /(N \cap K) \rightarrow N$. Therefore, jogoh $: M / K \rightarrow M$ is a nonzero homomorphism where $j: N \rightarrow M$ is the inclusion.

Recall that a module $M$ is hollow, provided every proper submodule of $M$ is small in $M$.

Corollary 2.9. (1) Let $M$ be a hollow module and $N<M$. Then $M$ is $N$-coretractable if and only if $M$ is coretractable.

(2) Let $M$ be a finitely generated module. Then $M$ is Rad(M)-coretractable if and only if $M$ is coretractable.

(3) Let $N$ be a semisimple submodule of $M$. Then $M$ is $N$-coretractable if and only if $M$ is coretractable.

(4) Let $M$ be a module. Then $M$ is Soc(M)-coretractable if and only if $M$ is coretractable.

Let $M$ be a module and $N$ a submodule of $M$. Following [15], $N$ is $\delta$-small in $M$ (denoted by $\left.N \ll_{\delta} M\right)$, in case $M=N+K$ with $M / K$ singular implies that $M=K$. Note that by definitions, every small submodule of $M$ is $\delta$-small in $M$. The sum of all $\delta$-small submodules of $M$ is denoted by $\delta(M)$. Also $\delta(M)$ is the reject of the class of all simple singular modules in $M$.

Proposition 2.10. Let $M$ be a module and $N$ be a proper $\delta$-small submodule of $M$. Then $M$ is $N$ coretractable if and only if $M$ is coretractable.

Proof. Let $M$ be $N$-coretractable and $K$ be a proper submodule of $M$. Suppose that $M \neq N+K$. Since $M$ is $N$-coretractable, there is a nonzero homomorphism $f: M /(N+K) \rightarrow M$. So that $f o \pi: M / K \rightarrow M$ is the required homomorphism where $\pi: M / K \rightarrow M /(N+K)$ is natural epimorphism. Otherwise, $M=N+K$. Now from [15, Lemma 1.2], there is a decomposition $M=Y \oplus K$ where $Y$ is a semisimple projective submodule of $N$. Therefore, there is a monomorphism from $M / K$ to $M$ since $K$ is a direct summand of $M$. It follows that $M$ is coretractable.

Proposition 2.11. Let $M$ be a module and $N$ be a proper submodule of $M$. If $M$ is $N$-coretractable and $M / N$ has a maximal submodule, then $\operatorname{Soc}(M) \neq 0$. In particular, if $M$ is finitely generated and $N$-coretractable, then $\operatorname{Soc}(M) \neq 0$.

Proof. Let $K / N$ be a maximal submodule of $M / N$. Then $K$ is a maximal submodule of $M$. So there is a nonzero homomorphism $h: M / K \rightarrow M$. It follows that $I m h$ is a simple submodule of $M$. This completes the proof.

The following is an immediate consequence of last proposition.

Corollary 2.12. Let $R$ be a ring such that every cyclic right $R$-module is coretractable relative to at least one of its submodules. Then $R$ is semi-Artinian.

Let $R$ be a ring. Then $R$ is called a right $V$-ring in case every simple right $R$-module is injective. As a generalization of $V$-rings, $R$ is a right generalized $V$-ring ( $G V$-ring for short), if every simple singular right $R$-module is injective ([11]). 
Proposition 2.13. Let $R$ be a ring and $M$ be an indecomposable right $R$-module with $\operatorname{Rad}(M) \neq M$. If each of the following statements holds, then $M$ is $\operatorname{Rad}(M)$-coretractable if and only if $M$ is simple.

(1) $R$ is a right $G V$-ring.

(2) $M$ is noncosingular.

Proof. (1) Let $M$ be $\operatorname{Rad}(M)$-coretractable. Then for each maximal submodule $K$ of $M$ there is a monomorphism $g: M / K \rightarrow M$. It follows that $I m g$ is a simple submodule of $M$. Then $I m g$ is either singular or projective. If $I m g$ is projective, then $K$ is a direct summand of $M$ and hence $K=0$ or $K=M$. So that $K=0$. If $I m g$ is singular, it will be injective as $R$ is right $G V$. Therefore, $\operatorname{Img}$ is a summand of $M$ and since $g \neq 0$ we conclude that $\operatorname{Img}=M$. In both cases, $M$ is simple. The converse is obvious.

(2) It follows from (1) and the fact that every homomorphic image of $M$ is noncosingular.

Corollary 2.14. Let $R$ be a right $V$-ring and $M$ an indecomposable right $R$-module. Then $M$ is coretractable if and only if $M$ is simple.

Following [8], a module $M$ is dual Rickart provided that for every $f \in \operatorname{End}(M), \operatorname{Im} f$ is a direct summand of $M$.

Remark 2.15. Let $M$ be an indecomposable dual Rickart module with $\operatorname{Rad}(M) \neq M$. Then $M$ is $(\operatorname{Rad}(M)-)$ coretractable if and only if $M$ is simple. Let $K$ be a maximal submodule of $M$. Then there is a monomorphism $g: M / K \rightarrow M$. Consider the endomorphism $h=g o \pi: M \rightarrow M$ where $\pi: M \rightarrow M / K$ is the natural epimorphism. Then Imh $=$ Img is a summand of $M$. So Img $=M$ as $M$ is indecomposable. It follows that $M$ is simple.

Proposition 2.16. Let $M$ be a module and $L$ a proper submodule of $M$ such that $L$ has a supplement $K$ in $M$. If $M$ is L-coretractable and $K$ is fully invariant in $M$, then $K$ is coretractable.

Proof. Let $K$ be a supplement of $L$ in $M$. Then $M=K+L$ and $K \cap L \ll K$. Let $N$ be a proper submodule of $K$. Then $N+L$ is a proper submodule of $M$. For if, $N+L=M$, by modular law $N+(K \cap L)=K$, which implies that $N=K$, a contradiction. Since $M$ is $L$-coretractable, there is a nonzero homomorphism $f: M /(N+L) \rightarrow M$. Since $K$ is a fully invariant submodule of $M$, we have $f \circ \pi(K) \subseteq K$ where $\pi: M \rightarrow M /(N+L)$ is the natural epimorphism. Now consider $h: K / N \rightarrow K$ by $h(x+N)=f(x+N+L)$ for every $x \in K$. It is not hard to verify that $h$ is well-defined. Now, there is $y \in M$ such that $y \notin N+L$ and $f(y+N+L) \neq 0$. Now there exists $k \in K$ and $l \in L$ such that $y=k+l$. It is easy to see that $h(k+N)=f(k+l+N+L)=f(y+L) \neq 0$. It follows that $h$ is nonzero.

Corollary 2.17. ([2, Proposition 2.5]) Every fully invariant direct summand of a coretractable module is coretractable.

Let $M$ be a module. Then $M$ is called a duo module provided every submodule of $M$ is fully invariant.

Corollary 2.18. Let $M$ be a duo module. If $M$ is coretractable relative to each direct summand of $M$, then every direct summand of $M$ is coretractable.

Proposition 2.19. Let $M=M_{1} \oplus \ldots \oplus M_{n}$ and $N<M$. If each $M_{i}$ is $N \cap M_{i}$-coretractable, then $M$ is $N$-coretractable. Especially a finite direct sum of coretractable modules is coretractable.

Proof. The proof is exactly similar to proof of [2, Proposition 2.6].

Proposition 2.20. Let $R$ be a right max ring and $M=\bigoplus_{i \in I} M_{i}$ be a direct sum of $N \cap M_{i}$-coretractable right $R$-modules where $N<M$. Then $M$ is $N$-coretractable. In particular, an arbitrary direct sum of coretractable right $R$-modules is coretractable.

Proof. Similar to the proof of [2, Proposition 2.7]. 
Let $M$ be an $R$-module. A submodule $K$ of $M$ is said to be dense in $M$ if, for any $y \in M$ and $0 \neq x \in M$, there exists $r \in R$ such that $x r \neq 0$ and $y r \in K$. Obviously, any dense submodule of $M$ is essential in $M$. From [7, Proposition 8.6], $K$ is dense in $M$ if and only if $\operatorname{Hom}_{R}(P / K, M)=0$ for every submodule $P \supseteq K$.

Remark 2.21. Let $M$ be a module and $N<M$. If $N$ is dense in $M$, then $M$ is not $N$-coretractable. In fact for a $N$-coretractable module $M$, we have $N$ is not dense in $M$. This follows from the fact that if $M$ is $N$-coretractable, then there is a nonzero homomorphism from $M / N$ to $M$.

Proposition 2.22. Let $M$ be a module and $N$ a proper submodule of $M$. If $M$ is quasi-injective or every proper submodule of $M$ is contained in a maximal submodule, then $M$ is $N$-coretractable if and only if every proper submodule of $M$ containing $N$ is not dense in $M$.

Proof. (1) Let $M$ be a quasi-injective module such that every proper submodule of $M$ containing $N$ is not dense in $M$. Suppose that $K$ is a proper submodule of $M$ containing $N$. Since $K$ is not dense in $M$, there is a $f: P / K \rightarrow M$ where $P$ is a submodule of $M$ containing $K$. It follows that $f o \pi: P \rightarrow M$ is a nonzero homomorphism where $\pi: P \rightarrow P / K$ is the natural epimorphism. Consider the inclusion homomorphism $j: P \rightarrow M$. Since $M$ is quasi-injective, there exists $h: M \rightarrow M$ such that $h o j=f o \pi$. By defining $\bar{h}: M / K \rightarrow M$ with $\bar{h}(m+K)=h(m)$ we conclude that $M$ is $N$-coretractable. Note that $\bar{h}$ is nonzero. Conversely, if $M$ is $N$-coretractable and $N \subseteq K<M$, then there is a homomorphism $g: M / K \rightarrow M$ which shows that $K$ is not dense in $M$.

(2) Suppose that every submodule of $M$ is contained in a maximal submodule of $M$. Let $N \subseteq K<M$. Then there is a maximal submodule $L$ of $M$ such that $K \leq L$. Since $L$ is not dense in $M$, there is a nonzero homomorphism $h: M / L \rightarrow M$. As $f: M / K \rightarrow M / L$ with $f(x+K)=x+L$ is a nonzero homomorphism, then hof is nonzero. It follows that $M$ is $N$-coretractable. The converse is the same as (1).

The following presents a characterization of $I$-coretractable rings.

Theorem 2.23. Let $R$ be a ring and $I$ be a proper right ideal of $R$. Then the following are equivalent:

(1) $R_{R}$ is I-coretractable;

(2) Every $n$-generated free right $R$-module is $I^{(n)}$-coretractable;

(3) For every right ideal $T \supseteq I$, $\operatorname{ann}_{l}(T) \neq 0$.

Proof. $\quad(1) \Leftrightarrow(2)$ Follows from Proposition 2.19.

$(1) \Rightarrow(3)$ Let $T$ be a right ideal of $R$ containing $I$. Since $R_{R}$ is $I$-coretractable, there is a nonzero homomorphism $f: R / T \rightarrow R$. Consider the endomorphism $g=f_{o} \pi: R \rightarrow R$ where $\pi$ is the natural epimorphism from $R$ to $R / T$. Then there is an element $0 \neq a \in R$ such that $g(x)=a x$. Let $y \in T$. Then $g(y)=a y=0$ as $T \subseteq K e r g$. This shows that $0 \neq a \in a n_{l}(T)$.

$(3) \Rightarrow(1)$ Let $T$ be a right ideal of $R$ containing $I$. Since $\operatorname{ann}_{l}(T) \neq 0$, there exists an element of $R$ such as $a$ that $a T=0$ and $a \neq 0$. Define $f: R / T \rightarrow R$ by $f(x+T)=a x$. It is easy to check that $f$ is an $R$-homomorphism and in particular $f \neq 0$.

Remark 2.24. Let $R$ be a ring and $I \leq R_{R}$ with ann $(I)=0$. Then $R_{R}$ is not $I$-coretractable. For example, let $R=\left[\begin{array}{cc}K & K \\ 0 & K\end{array}\right]$ be the ring of $2 \times 2$ upper triangular matrices over a field $K$. Let $I=\left[\begin{array}{cc}0 & K \\ 0 & K\end{array}\right]$ which is a right ideal of $R$. Then ann $_{l}(I)=0$. Hence, $R_{R}$ is not I-coretractable. In other words, $R / J(R)$ is coretractable relative to each of its ideals as $R / J(R)$ is a semisimple ring. Note that $J(R)=\left[\begin{array}{cc}0 & K \\ 0 & 0\end{array}\right]$.

Theorem 2.25. Let $R$ be a ring and $I$ be a proper two-sided ideal of $R$. Then the following statements are equivalent:

(1) $R_{R}$ is I-coretractable;

(2) Every simple right $R$-module that is annihilated by $I$ can be embedded in $R_{R}$. 
Proof. $\quad(1) \Rightarrow(2)$ Let $M \cong R / K$ be a simple right $R$-module such that $M I=0$. It follows that $I \subseteq K$. Since $R_{R}$ is $I$-coretractable, there is a nonzero homomorphism $f: R / K \rightarrow R$.

$(2) \Rightarrow(1)$ Let $T$ be a right ideal of $R$ containing $I$. Now there exists a right maximal ideal $K$ of $R$ such that $I \subseteq T \subseteq K$. Consider the simple right $R$-module $M=R / K$. Since $M I=0$, there is a nonzero homomorphism $g: R / K \rightarrow R$ by assumption. As $T$ is a submodule of $K$, there exists $f: R / T \rightarrow R / K$ defined by $f(x+T)=x+K$. Hence $g o f$ is the desired homomorphism.

For a ring $R$, Theorem 2.25 implies that $R_{R}$ is $(J(R)$-)coretractable if and only if $R$ is a right Kasch ring.

In [2, Proposition 4.4], it is shown that if $R$ is a von Neumann regular ring then $R$ is right Kasch if and only if $R$ is semisimple. In the following we shall investigate a more general version.

Proposition 2.26. Let $R$ be a right $G V$-ring. Then the following are equivalent:

(1) $R$ is right Kasch;

(2) $R$ is semisimple.

Proof. $\quad(1) \Rightarrow(2)$ Let $R$ be right Kasch. So $R_{R}$ is $J(R)$-coretractable. Now suppose that $K$ is an arbitrary maximal right ideal of $R$. Then there is a monomorphism $g: R / K \rightarrow R$. It follows that $R / K \cong I m g$ is a simple right $R$-module. So, Img is either singular or projective. In first case Img should be injective as $R$ is right $G V$. Therefore, Img is a direct summand of $R_{R}$. Now Img is singular projective which implies that $I m g=0$, a contradiction. So that $I m g$ and hence every simple right $R$-module will be projective. This shows that $R$ is semisimple.

$(2) \Rightarrow(1)$ It is obvious.

Corollary 2.27. Let $R$ be a right $V$-ring. Then $R$ is a Kasch ring if and only if $R$ is semisimple.

Example 2.28. (1) Let $R=\left[\begin{array}{cc}K & K \\ 0 & K\end{array}\right]$ where $K$ is a field. Then $J(R)=\left[\begin{array}{cc}0 & K \\ 0 & 0\end{array}\right]$. It is easy to check that $R$ is a semilocal ring as $R / J(R) \cong K \times K$ which is a semisimple ring. Now by [3, Exercise 10, Page 113], $\operatorname{Soc}\left({ }_{R} R\right)=\left[\begin{array}{cc}K & K \\ 0 & 0\end{array}\right]$. However, $\operatorname{Soc}\left(R_{R}\right)=\left[\begin{array}{cc}0 & K \\ 0 & K\end{array}\right]$. Set $m_{1}=\operatorname{Soc}\left({ }_{R} R\right)$ and $m_{2}=\operatorname{Soc}\left(R_{R}\right)$. Then both $m_{1}$ and $m_{2}$ are maximal left and right ideals of $R$. A quick calculation shows that ann $n_{l}\left(m_{1}\right)=m_{2}$, ann $_{l}\left(m_{2}\right)=0$, ann $n_{r}\left(m_{1}\right)=0$ and ann $_{r}\left(m_{2}\right)=m_{1}$. Now by Theorem 2.23, $R_{R}$ is $m_{1}$-coretractable while $R_{R}$ is not $m_{2}$-coretractable. Also left version of Theorem 2.23, implies that ${ }_{R} R$ is $m_{2}$-coretractable but it is not $m_{1}$-coretractable. Since the simple right $R$-module $R / m_{2}$ can not be embedded in $R_{R}$ and the simple left $R$-module $R / m_{1}$ can not be embedded in ${ }_{R} R$, the ring $R$ is neither right Kasch nor left Kasch (note that since $R$ is right $G V$ which is not a $V$-ring, it can not be Kasch from Proposition 2.26).

(2) Let $K$ be a division ring and $R=\left\{A=\left[\begin{array}{llll}a & 0 & b & c \\ 0 & a & 0 & d \\ 0 & 0 & a & 0 \\ 0 & 0 & 0 & e\end{array}\right] \mid a, b, c, d, e \in K\right\}$. Then $J(R)=\{A \in$ $R \mid a=0=e\}, \operatorname{Soc}\left(R_{R}\right)=\operatorname{ann}_{l}(J(R))=\{A \in R \mid a=0\}, \operatorname{Soc}\left({ }_{R} R\right)=\operatorname{ann}_{r}(J(R))=J(R)$. Since $R / J(R) \cong K \times K, R$ is a semilocal ring. Now $\operatorname{Soc}\left(R_{R}\right)=\{A \in R \mid a=0\}$ and $\operatorname{Soc}\left({ }_{R} R\right)=J(R)$. From [7, Example 8.29], $\operatorname{Soc}\left(R_{R}\right)$ is a left and right maximal ideal of $R$. Since ann $\sin _{r}\left(\operatorname{Soc}\left(R_{R}\right)\right)=\{A \in R \mid$ $a=e=0\}=J(R) \neq 0$, it follows from [7, Corollary 8.28], $R / \operatorname{Soc}\left(R_{R}\right)$ can be embedded in ${ }_{R} R$ (see also Theorem 2.23). Therefore, ${ }_{R} R$ is $\operatorname{Soc}\left(R_{R}\right)$-coretractable while ${ }_{R} R$ is not $\operatorname{Soc}\left({ }_{R} R\right)$-coretractable (see also Corollary 2.9). Now an easy computation shows that ann $n_{l}\left(\operatorname{Soc}\left(R_{R}\right)\right)=\{A \in R \mid a=c=d=e=0\} \neq 0$. So $R / \operatorname{Soc}\left(R_{R}\right)$ can be embedded in $R_{R}$ by [7, Corollary 8.28]. As Soc $\left(R_{R}\right)$ is a maximal right ideal of $R$, then $R_{R}$ is $\operatorname{Soc}\left(R_{R}\right)$-coretractable. Also from [7, Example 8.29], $R$ is a right Kasch ring while it is not a left Kasch ring.

(3) Let $K$ be a field and $R=\prod_{i=1}^{\infty} K$. It is well-known that $R$ is a Von Neumann regular $V$-ring. Consider the ideal $T_{i}=K \times K \times \ldots \times K \times 0 \times K \times K \times \ldots$ It is clear that $T_{i}$ for each $i \in \mathbb{N}$ is a maximal 
ideal of $R$. It is easy to see that ann $\left(T_{i}\right)=0 \times 0 \times \ldots \times 0 \times K \times 0 \times \ldots$ which is nonzero. Therefore, from Theorem 2.23, $R$ is $I$-coretractable for each $I \subseteq T_{i}$. Now consider the ideal $L=\bigoplus_{i=1}^{\infty} K$ of $R$. Then ann $(L)=0$ and of course ann $(m)=0$ for every maximal ideal $m$ of $R$ containing $L$. Hence the simple $R$-module $R / m$ can not be embedded in $R$ (see [7, Corollary 8.28]). Therefore, $R$ is not coretractable relative to L. This means that $R$ is not a Kasch ring.

Proposition 2.29. Let $R$ be a ring and I a right ideal of $R$ such that every free right $R$-module $R^{(A)}$ is $\left(I^{(A)}\right)$-coretractable. Then for every right $R$-module $M$ with $I \subseteq a n n_{r}(M), \operatorname{Hom}_{R}(M, R) \neq 0$.

Proof. Let $M$ be a right $R$-module such that $I \subseteq a n n_{r}(M)$. Then there is a free right $R$-module $F$ and a submodule $K$ of $F$ such that $M \cong F / K$. Since $M I=0$, we have $I^{(A)} \subseteq K$ where $A$ is an indexed set. By assumption, there is a nonzero homomorphism $f: F / K \rightarrow F$. Then the homomorphism $\pi \circ f: M \rightarrow R$ is the required one where $\pi: F \rightarrow R$ is the natural epimorphism.

Proposition 2.30. Let $R$ be a ring having a radical right $R$-module $M$ with $M I \neq M$ where $I \leq R_{R}$. If for every right ideal $T$ of $R, \operatorname{Rad}(T) \neq T$, then there is a free right $R$-module $R^{(A)}$ which is not $I^{(A)}$-coretractable.

Proof. Let $\operatorname{Rad}(M)=M$ such that $M I$ is a proper submodule of $M$. There exists a free right $R$-module $F=R^{(A)}$ and a submodule $K$ of $F$ such that $M / M I \cong F / K$. Being $M$ radical implies that $M / M I$ is radical. So, $\operatorname{Hom}_{R}(M / M I, R)=0$. Since $(F / K) I=0, I^{(A)} \subseteq K$. It follows that $\operatorname{Hom}_{R}(F / K, F)=0$ which implies $F$ is not $I^{(A)}$-coretractable.

Proposition 2.31. Let $R$ be a right max ring and $I \leq R_{R}$ such that every cyclic $R$-module $N$ is $N I$ coretractable. Then every right $R$-module $M$ is $M I$-coretractable. In particular, if $R$ is a (semiperfect) right perfect ring with all cyclic right $R$-modules coretractable, then every (finitely generated) right $R$ module is coretractable.

Proof. Let $M$ be a right $R$-module. Suppose that $K$ is a proper submodule of $M$ containing $M I$. Since $R$ is a right max ring, $K$ is contained in a maximal submodule $L$ of $M$. For every $x \in M \backslash L$, we know $M / L \cong x R /(x R \cap L)$ as $x R+L=M$. Note that $M I \subseteq L$. So that $(x R /(x R \cap L)) I=0$. It follows that $(x R) I \subseteq x R \cap L$. Being $x R$ a $(x R) I$-coretractable module implies that $\operatorname{Hom}_{R}(x R /(x R \cap L), x R) \neq 0$. Hence there is a nonzero homomorphism $f: M / L \rightarrow M$. Therefore, $H_{o m}(M / K, M) \neq 0$ as $K \subseteq L$.

\section{References}

[1] A. N. Abyzov, A. A. Tuganbaev, Retractable and coretractable modules, J. Math. Sci. 213(2) (2016) $132-142$.

[2] B. Amini, M. Ershad, H. Sharif, Coretractable modules, J. Aust. Math. Soc. 86(3) (2009) 289-304.

[3] F. W. Anderson, K. R. Fuller, Rings and Categories of Modules, Springer-Verlog, New York, 1992.

[4] N. O. Ertaş, D. K. Tütüncü, R. Tribak, A variation of coretractable modules, Bull. Malays. Math. Sci. Soc. 41(3) (2018) 1275-1291.

[5] S. M. Khuri, Endomorphism rings and lattice isomorphisms, J. Algebra 56(2) (1979) 401-408.

[6] S. M. Khuri, Nonsingular retractable modules and their endomorphism rings, Bull. Aust. Math. Soc. 43(1) (1991) 63-71.

[7] T. Y. Lam, Lectures on Modules and Rings, Springer-Verlag, New York, 1999.

[8] G. Lee, S. T. Rizvi, C. S. Roman, Dual Rickart modules, Comm. Algebra 39(11) (2011) 4036-4058.

[9] S. H. Mohamed, B. J. Müller, Continuous and Discrete Modules, London Math. Soc. Lecture Notes Series 147, Cambridge, University Press, Cambridge, 1990.

[10] A. R. M. Hamzekolaee, A generalization of coretractable modules, J. Algebraic Syst. 5(2) (2017) $163-176$. 
[11] R. Wisbauer, Foundations of Module and Ring Theory, Gordon and Breach, Philadelphia, 1991.

[12] J. M. Zelmanowitz, Correspondences of closed submodules, Proc. Amer. Math. Soc. 124(10) (1996) $2955-2960$.

[13] J. Žemlička, Completely coretractable rings, Bull. Iranian Math. 39(3) (2013) 523-528.

[14] Z. Zhengping, A lattice isomorphism theorem for nonsingular retractable modules, Canad. Math. Bull. 37(1) (1994) 140-144.

[15] Y. Zhou, Generalizations of perfect, semiperfect, and semiregular rings, Algebra Colloq. 7(3) (2000) $305-318$. 